\title{
COVID-19 Screening By Anti-SARS-CoV-2 Antibody Seropositivity: Clinical And Epidemiological Characteristics, Comorbidities, And Food Intake Quality
}

\section{G Macedo-0jeda}

Department of Public Health, University Center of Health Sciences (CUCS), University of Guadalajara, Guadalajara, Mexico

JF Muñoz-Valle

Institute of Research in Biomedical Sciences, University Center of Health Sciences (CUCS), University of Guadalajara, Guadalajara, México.

\section{P Yokogawa-Teraoka}

Department of Social Sciences, University Center for Health Sciences (CUCS), University of Guadalajara, Guadalajara, México

\section{AC Machado-Sulbarán}

Institute for Research in Childhood and Adolescent Cancer (INICIA), University Center for Health Sciences (CUCS), University of Guadalajara, Guadalajara, México

\section{MG Loza-Rojas}

Department of Applied Clinical Nursing, University Center for Health Sciences (CUCS), University of Guadalajara, Guadalajara, México

\section{AC García Arredondo}

Department of Applied Clinical Nursing, University Center for Health Sciences (CUCS), University of Guadalajara, Guadalajara, México

\section{R Tejeda-Constantini}

Institutional System of Safety, Health and Environment (SISSMA), University of Guadalajara, Guadalajara, México

\section{AN Vega-Magaña}

Emerging and Reemerging Diseases Diagnostic Laboratory (LaDEER), University Center for Health Sciences (CUCS), University of Guadalajara, Guadalajara, México

\section{G González-Estevez}

Institute of Research in Biomedical Sciences, University Center of Health Sciences (CUCS), University of Guadalajara, Guadalajara, México.

\section{García-Chagollán}

Institute of Research in Biomedical Sciences, University Center of Health Sciences (CUCS), University of Guadalajara, Guadalajara, México. 


\section{JS Zepeda-Nuño}

Department of Microbiology and Pathology, Pathology Laboratory, University Center for Health Sciences (CUCS), University of Guadalajara, Guadalajara, México

J Hernández-Bello ( $\square$ jorge89_5@hotmail.com )

COVID-19 Health Situation Room, University of Guadalajara, Guadalajara, México

\section{Research Article}

Keywords: COVID-19, serological testing, clinical characteristics, food intake quality.

Posted Date: January 26th, 2021

DOI: https://doi.org/10.21203/rs.3.rs-154551/v1

License: (c) (i) This work is licensed under a Creative Commons Attribution 4.0 International License.

Read Full License 


\section{Abstract}

Background: Developing countries have reported lower molecular diagnostic testing levels due to a lack of infrastructure and resources. Therefore, antibody tests represent an alternative to detect exposure to SARS-CoV-2 and analyze possible risk factors in them. This study aimed to describe and compare the clinical and epidemiological characteristics and the quality of food intake between individuals with a positive or negative test to antibodies against SARS-CoV-2.

Methods: Mexican individuals of diverse ages and sex, that attended to detect anti-SARS-CoV-2 antibody seropositivity from July to November 2020 were included. A survey was applied to identify the clinical and epidemiological characteristics and food intake quality in negative and positive individuals for antiSARS-CoV-2 antibodies. These variables were compared using the Chi-square test, Kruskal Wallis, Fisher's exact test, or t-student.

Results: In this study, a total of 1,799 individuals participated; $42 \%$ were positive for SARS-CoV-2. It was found that seropositive subjects were older and that diabetes was more prevalent in them $(p<0.01)$. No differences were identified in the blood type nor vaccination against influenza between seronegative and seropositive cases. Cough, respiratory distress, muscle pain, joint pain, and anosmia were more prevalent in seropositive cases than in those seronegative $(p<0.0001)$. The $\lg M$ or $\lg M+\lg G$ seropositive cases presented anosmia more frequently than those with only IgG antibodies $(p<0.05)$. The dietary patterns and individual food intake habits were similar in both groups, except for the most consumed type of fat $(p=0.006)$. Seronegative cases showed to consume monounsaturated fats more frequently than other types of fats, whereas seropositive subjects have more regular consumption of polyunsaturated fats.

Conclusion: This study confirms the association between being anti-SARS-CoV-2 seropositive with older age and the presence of diabetes mellitus. Furthermore, it suggests muscle and joint pain could also be considered major criteria for discriminating patients with suspected COVID-19 seropositivity. Moreover, the results suggested polyunsaturated fats as a possible new risk factor associated with COVID-19, and anosmia as a marker of active disease. This finding will be of interest to investigate in further studies regarding the type of fat consumed and the risk of infection by SARS-CoV- 2 .

\section{Background}

Coronavirus disease 2019 (COVID-19) is a current pandemic disease caused by the severe acute respiratory syndrome coronavirus 2 (SARS-CoV-2) infection [1]. Considerable efforts have been made to contain this disease; however, the pandemic has continued active in many countries, displaying infections with varying degrees of clinical manifestations. With the global increase of COVID-19 cases, the accurate and early detection of positive cases is necessary to manage them better and stop contagion [2].

Quantitative reverse transcription PCR (RT-qPCR) testing is the current gold standard for diagnosing SARS-CoV-2 infection; however, technical issues limit its utilization for large-scale screening in developing 
countries, due to the insufficient infrastructure in molecular biology. In these countries, serological immunoglobulin $\mathrm{M}(\mathrm{IgM}) / \mathrm{lgG}$ testing is an alternative for detecting SARS-CoV-2 exposure [3].

A recent meta-analysis aimed to assess the diagnostic accuracy of antibody tests for detecting active or previous SARS-CoV-2 infection [4]. This analysis evaluated 54 study cohorts with 8,526 cases of SARSCoV-2 infection. They reported low sensitivity for pooled results for $\lg G$, $\lg M$, IgA during the first week since onset of symptoms $(<30.1 \%)$, rising in the second week $(72.2 \%)$, and reaching their highest values in the third week (91.4\%) [4]. The sensitivity reported by this study was mainly evaluated in hospitalized patients, so its reproducibility is unclear in cohorts of outpatients with milder and asymptomatic COVID19 , in whom lower antibody levels have been suggested [5].

The clinical relevance and use of serological testing are still an open debate; for this reason, this study aimed to describe the clinical and epidemiological characteristics of Mexican individuals attending a COVID-19 diagnostic module for a serologic test due to suspected SARS-CoV-2 infection. We also analyzed the relationship between $\mathrm{lgG} / \mathrm{lgM}$ expression and the onset of clinical symptoms and some previously suggested susceptibility/protection factors for COVID-19, such as blood type [6-9] and influenza vaccination status [10-13]. Moreover, we evaluated the food intake quality among the study individuals as substantial stressors, as inadequate nutrition can lead to long-effects affecting health and contribute to comorbidities associated with a higher SARS-CoV-2 infection risk [14].

\section{Methods}

\section{Design and participants}

Cross-sectional study. All the individuals who agreed to participate, Mexicans of any age and sex, that attended a COVID-19 diagnostic module to detect anti-SARS-CoV-2 antibody seropositivity from July to November 2020, were included.

The University of Guadalajara installed laboratories to carry out molecular tests and modules for serological tests to detect SARS-CoV-2 infections. It also established a 24/7 call-center for managing testing appointments. The service was provided to the general population, free of charge. An algorithm (Figure 1) was used to determine the eligibility of every subject. The criteria included five emergency criteria, 23 signs and symptoms, and 17 risk factors.

\section{The setting of the study, materials, and process}

The participants attended the COVID-19 diagnostic module installed at the University of Guadalajara, México, where they were invited to participate in the study. All subjects that accepted to participate signed informed consent. If individuals were under 18 years old (legal age of majority in Mexico), their parents or guardians signed informed consent forms, as long as the minor agreed.

Antibody tests were carried out according to the test manufacturer's instructions (Test Cellex COVID 19 qSARS-CoV-2 lgG / IgM), in a specially conditioned location, following the indications of the safety sheets 
for each chemical substance used, and the recommendations provided for the handling and final disposal of material and reagents indicated in the test manual.

The staff verified that the patients wore their masks correctly and that the previously trained care personnel wore appropriate personal protective equipment. Once the test was done, the participants completed a survey that included two sections while they waited for their result (approximately 10 minutes). The first section consists of 14 questions to identify the participant comorbidities, clinical, and epidemiological characteristics; the second section includes 14 items of the Mini-Survey to Evaluate Food Intake Quality (Mini-ECCA v.2), previously validated [15].

After it was completed, the participants received their test results. This result was written in the survey, that was subsequently placed in a container, where it remained for two weeks before being captured, to avoid possible contamination due to the virus's presence on the surface of the material used to answer it.

\section{Statistical analysis}

Demographic characteristics, comorbidities, risk contacts, and symptoms were compared between negative and positive individuals for anti-SARS-CoV-2 antibodies, using the Chi-square test, Kruskal Wallis, Fisher's exact test, or t-student. In addition, symptoms in positive individuals were compared according to seropositivity to anti-SARS-CoV-2 antibodies (IgM, IgG, $\operatorname{lgM}+\lg G)$, using the same statistical tests.

A cluster analysis was performed using Ward's method for non-standardized variables to identify dietary patterns. Subsequently, a simple analysis of variance (ANOVA) was performed for each item of the questionnaire to compare the groups identified in the cluster analysis. Then, a discriminant analysis was performed to verify that the dietary patterns identified in the cluster analysis had significant differences between them. Finally, individual food intake habits and dietary patterns were compared using the Chisquare test.

Statistical analyses were conducted using the Statistical Package for the Social Sciences (SPSS Statistics for Windows, Version 17.0. Chicago: SPSS Inc.).

\section{Results}

\section{Clinical and epidemiological characteristics of the study participants}

There were 1,799 individuals with a serology test result (positive or negative) during the study period; $42 \%$ of cases were positives for SARS-CoV-2 (anti-SARS-CoV-2 IgM, IgG, or IgM+lgG seropositivity), and $58 \%$ of them were negative (Table 1). Seropositive cases were older than those seronegative $(p=0.0001)$ : median 37 (IQR: 28-49) years old vs. 34 years (IQR: 25-46), respectively. From the 703 seropositive cases and 979 seronegative cases with information on age, the age range of seropositive cases was dominantly $21-30$ years old (27.03\%), followed by the group of 31-40 years old (26.74\%); this finding was statistically different from what was observed in seronegative cases $(p=0.002)$. 
A higher proportion of seropositive (58.99\%) and seronegative (56.57\%) individuals were women. Moreover, most of the individuals (seropositive or seronegative) reported a previous record of vaccination against influenza (>50\%). Regarding comorbidities, overweight was the most prevalent in both study groups ( $19.84 \%$ in seropositive cases and $17.35 \%$ in those seronegative, $p=0.304)$; however, diabetes was most prevalent in seropositive cases in comparison with those seronegative $(9.39 \%$ vs. $5.56 \%$, respectively, $p=0.002$ ). Seropositive cases declared more symptoms than those seronegative (mean \pm SD: $4.489 \pm 2.409$ vs. $3.810 \pm 2.423$, respectively, $p=0.008$ ).

Blood type did not show differences between both study groups $(p=0.403)$. On the other hand, seropositive cases declared less knowledge about having contact with a patient with COVID-19 before undergoing the serological test (seropositive cases $67.20 \%$ vs. seronegative cases $73.63 \%, p=0.003$ ). However, seropositive cases had more days of contact with COVID-19 patients pre-test (median 12 days [IQR: 7-17] vs. 10 days [IQR: 6-15], respectively, $p=0.0023$ ). In both study groups, the contact with SARSCoV-2 suspects was at home ( $46.56 \%$ in seropositive cases, and $47.17 \%$ in seronegative cases, $p=0.798$, data not shown).

Table 1. Demographic characteristics, comorbidities, and risk contacts of individuals negative or positive to anti-SARS-CoV-2 antibodies 


\begin{tabular}{|c|c|c|c|}
\hline \multirow[t]{2}{*}{ Variables } & $\begin{array}{l}\text { Positive for anti-SARS- } \\
\text { CoV-2 antibodies }\end{array}$ & $\begin{array}{l}\text { Negative for anti-SARS- } \\
\text { CoV-2 antibodies }\end{array}$ & $\begin{array}{c}\text { P- } \\
\text { value }^{\mathrm{a}}\end{array}$ \\
\hline & $\mathrm{N}=756$ & $\mathrm{~N}=1043$ & \\
\hline Age (years) Median (P25th-P75th) & $37(28-49)$ & $34(25-46)$ & $0.0001^{\mathrm{b}}$ \\
\hline $0-20$ & $40(5.69)$ & $89(9.09)$ & \multirow[t]{6}{*}{0.002} \\
\hline $21-30$ & $190(27.03)$ & $325(33.20)$ & \\
\hline $31-40$ & $188(26.74)$ & $236(24.11)$ & \\
\hline $41-50$ & $126(17.92)$ & $153(15.63)$ & \\
\hline $51-60$ & $100(14.22)$ & $121(12.36)$ & \\
\hline$>60$ & $59(8.39)$ & $55(5.62)$ & \\
\hline Sex: Female/Male ${ }^{\mathrm{n}, \%}$ & $446(58.99) / 310(41.01)$ & $590(56.57) / 453(43.43)$ & 0.304 \\
\hline Vaccinated against influenza $\mathrm{n}, \%$ & 385 (50.93) & 541 (51.87) & 0.693 \\
\hline \multicolumn{4}{|l|}{ Comorbidities $^{\mathrm{n}, \%}$} \\
\hline Overweight & $150(19.84)$ & $181(17.35)$ & 0.179 \\
\hline Obesity & $69(9.13)$ & $83(7.96)$ & 0.379 \\
\hline Hypertension & $87(11.51)$ & $109(10.45)$ & 0.477 \\
\hline Diabetes & $71(9.39)$ & $58(5.56)$ & 0.002 \\
\hline Immunodeficiency & $8(1.06)$ & $10(0.96)$ & $0.834^{\mathrm{C}}$ \\
\hline Number of comorbidities & $4.489 \pm 2.409$ & $3.810 \pm 2.423$ & $0.008^{d}$ \\
\hline \multicolumn{4}{|l|}{ Blood group $\mathrm{n}, \%$} \\
\hline $\mathrm{A}(\mathrm{Rh}+$ or $\mathrm{RH}-)$ & 215 (28.44) & $300(28.76)$ & \multirow[t]{5}{*}{0.403} \\
\hline $\mathrm{B}(\mathrm{Rh}+$ or $\mathrm{RH}-)$ & $61(8.07)$ & $94(9.01)$ & \\
\hline $\mathrm{AB}(\mathrm{Rh}+$ or $\mathrm{RH}-)$ & $19(2.51)$ & $32(3.07)$ & \\
\hline $\mathrm{O}(\mathrm{Rh}+\mathrm{or} \mathrm{RH}-)$ & $377(49.87)$ & $528(50.62)$ & \\
\hline Unknown & $84(11.11)$ & 89 (8.53) & \\
\hline \multicolumn{4}{|l|}{ Risk contact } \\
\hline Contact with COVID-19 patient n, \% & $508(67.20)$ & 768 (73.63) & 0.003 \\
\hline $\begin{array}{l}\text { Days of contact with COVID-19 patients } \\
\text { pre-test Median (P25th-P75th) }\end{array}$ & $12(7-17)$ & $10(6-15)$ & $0.0023^{b}$ \\
\hline
\end{tabular}

*Anti-SARS-CoV-2 IgM, IgG, or IgM+ IgG seropositivity; ${ }^{\mathrm{a} C h i-s q u a r e, ~}{ }^{\mathrm{b}}$ Kruskal-Wallis, ${ }^{\mathrm{c}}$ Fisher's exact test; ${ }^{d}$ t-student. Bold text indicates a statistically significant difference with a $p$-value less than 0.05 .

\section{Symptoms of the study participants}

The symptoms findings in seropositive cases and seronegative cases are shown in Table 2. Seropositive cases showed more days of symptom onset than seronegative cases (median 10 days [IQR: 7-14] vs. 8 days [IQR: 5-11], respectively, $p<0.0001)$. Similarly, seropositive cases declared a greater number of symptoms than seronegative cases (median 5 symptoms [IQR: 3-6] vs. 4 symptoms [IQR: 2-6], respectively, $\mathrm{p}<0.0001)$. 
The most prevalent symptom in both study groups was headache $(67.20 \%$ in seropositive cases and $65.96 \%$ in seronegative cases, $p=0.585$ ). On the other hand, hypogeusia, diarrhea, vomiting, upset stomach, pain chest, fatigue, and dizziness were the less frequent symptoms in both study groups (prevalency $\leq 6 \%$ ). There were statistically significant differences in the frequency of cough, respiratory distress, muscle and joint pain, and anosmia between both study groups, all of which were most frequent in seropositive cases $(p<0.0001)$.

\section{Table 2. Symptoms of individuals negative or positive to anti-SARS-CoV-2 antibodies}

\begin{tabular}{|c|c|c|c|}
\hline \multirow[t]{2}{*}{ Variables } & $\begin{array}{l}\text { Positive for anti-SARS-CoV-2 } \\
\text { antibodies }\end{array}$ & $\begin{array}{c}\text { Negative for anti-SARS-CoV-2 } \\
\text { antibodies }\end{array}$ & P-value \\
\hline & $\mathrm{N}=756$ & $\mathrm{~N}=1043$ & \\
\hline $\begin{array}{l}\text { Days of symptom onset }{ }^{\text {Median }} \\
\text { (P25th-P75th) }\end{array}$ & $10(7-14)$ & $8(5-11)$ & $<0.0001$ \\
\hline $\begin{array}{l}\text { Number of symptoms Median } \\
\text { (P25th-P75th) }\end{array}$ & $5(3-6)$ & $4(2-6)$ & $<0.0001$ \\
\hline Asymptomatic n, \% & $47(6.22)$ & $142(13.61)$ & $<0.0001$ \\
\hline Headache ${ }^{n, \%}$ & $508(67.20)$ & 688 (65.96) & 0.585 \\
\hline Cough $\mathrm{n}, \%$ & $437(57.80)$ & $481(46.12)$ & $<0.0001$ \\
\hline Fever $^{n, \%}$ & $246(32.54)$ & 307 (29.43) & 0.159 \\
\hline Respiratory distress ${ }^{\mathrm{n}, \%}$ & $194(25.66)$ & $176(16.87)$ & $<0.0001$ \\
\hline $\begin{array}{l}\text { runny nose, nasal congestion, } \\
\text { sneezing } n, \%\end{array}$ & $384(50.79)$ & $559(53.60)$ & 0.240 \\
\hline Muscle pain ${ }^{\mathrm{n}, \%}$ & $418(55.29)$ & $481(46.12)$ & $<0.0001$ \\
\hline Joint pain ${ }^{\mathrm{n}, \%}$ & $285(37.70)$ & $306(29.34)$ & $<0.0001$ \\
\hline Sore throat ${ }^{\mathrm{n}, \%}$ & $374(49.47)$ & $546(52.35)$ & 0.228 \\
\hline Anosmia $\mathrm{n}, \%$ & $453(59.92)$ & $305(29.24)$ & $<0.0001$ \\
\hline Hypogeusia ${ }^{\mathrm{n}, \%}$ & $39(5.16)$ & $27(2.59)$ & 0.004 \\
\hline $\begin{array}{l}\text { Diarrhea, vomiting, upset } \\
\text { stomach }^{\text {n, \% }}\end{array}$ & 37 (4.89) & $63(6.04)$ & 0.295 \\
\hline Chest pain ${ }^{n, \%}$ & $5(0.66)$ & $12(1.15)$ & $0.290 \mathrm{c}$ \\
\hline Fatigue $^{\mathrm{n}, \%}$ & $9(1.19)$ & $11(1.05)$ & $0.786^{\mathrm{c}}$ \\
\hline Dizziness ${ }^{\mathrm{n}, \%}$ & $5(0.66)$ & $12(1.15)$ & $0.290^{\mathrm{C}}$ \\
\hline
\end{tabular}

*Anti-SARS-CoV-2 IgM, IgG, or IgM+ IgG seropositivity. Bold text indicates a statistically significant difference with a p-value less than 0.05

\section{Symptoms according to seropositivity pattern to anti-SARS-CoV-2 antibodies}

The frequency of symptoms reported among individuals with anti-SARS-CoV-2 IgM, IgG, or IgM+lgG seropositivity was similar (Table 3). However, those who presented IgM+lgG reported cough more frequently $(61.6 \%)$ than those who only presented $\lg M(47.5 \%)(p=0.007)$. Moreover, anosmia was more frequent in those who presented $\operatorname{lgM}+\operatorname{lgg}(61.6 \%)$ than in those who presented $\lg G(49.6 \%)(p=0.034)$. 
Table 3. Symptoms according to seropositivity to anti-SARS-CoV-2 antibodies

\begin{tabular}{|c|c|c|c|c|}
\hline Variables & $\begin{array}{l}\text { Anti-SARS-CoV-2 IgM } \\
\text { seropositivity }\end{array}$ & $\begin{array}{l}\text { Anti-SARS-CoV-2 IgG } \\
\text { seropositivity }\end{array}$ & $\begin{array}{c}\text { Anti-SARS-CoV-2 IgM + } \\
\text { IgG seropositivity }\end{array}$ & $\begin{array}{c}\mathrm{P}- \\
\text { value }\end{array}$ \\
\hline \multirow{3}{*}{$\begin{array}{l}\text { Days of symptom onset } \\
\text { (Median, P25th-P75th) }\end{array}$} & $\mathrm{N}=69$ & $\mathrm{~N}=66$ & $\mathrm{~N}=270$ & \\
\hline & $9.00(7.00-11.00)$ & $10(7.75-13.25)$ & $10.00(7.00-15.00)$ & $0.086^{\mathrm{b}}$ \\
\hline & $\mathrm{N}=118$ & $\mathrm{~N}=125$ & $\mathrm{~N}=513$ & \\
\hline $\begin{array}{l}\text { Number of symptoms } \\
\text { (Median, P25th-P75th) }\end{array}$ & $4.50(3.00-6.00)$ & $4.00(2.00-6.00)$ & $5.00(3.00-6.50)$ & $0.088^{\mathrm{b}}$ \\
\hline Headache ${ }^{\mathrm{n}, \%}$ & $82(69.5)$ & $81(64.8)$ & $345(67.3)$ & 0.750 \\
\hline Cough $^{\text {n, \% }}$ & $56(47.5)$ & $65(52.0)$ & $316(61.6)$ & 0.007 \\
\hline Fever $n, \%$ & $34(28.8)$ & $33(26.4)$ & $179(34.9)$ & 0.122 \\
\hline Respiratory distress $^{n,} \%$ & $24(20.3)$ & $23(18.4)$ & $147(28.7)$ & 0.022 \\
\hline $\begin{array}{l}\text { Runny nose, nasal } \\
\text { congestion, sneezing } \mathrm{n}, \%\end{array}$ & $65(55.1)$ & $61(48.8)$ & $258(50.3)$ & 0.576 \\
\hline Muscle pain ${ }^{\text {n, } \%}$ & $69(58.5)$ & $66(52.8)$ & $283(55.2)$ & 0.672 \\
\hline Joint pain ${ }^{n, \%}$ & $48(40.7)$ & $40(32.0)$ & $197(38.4)$ & 0.324 \\
\hline Sore throat ${ }^{n, \%}$ & $57(48.3)$ & $62(49.6)$ & $255(49.7)$ & 0.969 \\
\hline Anosmia ${ }^{\text {n, \% }}$ & $75(63.6)$ & $62(49.6)$ & $316(61.6)$ & 0.034 \\
\hline Hypogeusia n, \% & $11(9.3)$ & $6(4.8)$ & $22(4.3)$ & 0.082 \\
\hline $\begin{array}{l}\text { Diarrhea, vomiting, upset } \\
\text { stomach } \text { n, \% }\end{array}$ & $7(5.9)$ & $2(1.6)$ & $28(5.5)$ & 0.174 \\
\hline Chest pain ${ }^{n} \%$ & $1(0.8)$ & $1(0.8)$ & $3(0.6)$ & $0.659^{\mathrm{C}}$ \\
\hline Fatigue $^{\mathrm{n}, \%}$ & $1(0.8)$ & $4(3.2)$ & $4(0.8)$ & $0.092^{\mathrm{C}}$ \\
\hline Dizziness $^{\text {n, \% }}$ & $1(0.8)$ & $1(0.8)$ & $3(0.6)$ & $0.659^{\mathrm{C}}$ \\
\hline
\end{tabular}

${ }^{a}$ Chi-square, ${ }^{b}$ Kruskal-Wallis, ${ }^{c}$ Fisher's exact test, Bold text indicates a statistically significant difference with a p-value less than 0.05 .

\section{Food intake quality}

Three dietary patterns were identified by the cluster analysis (healthy, unhealthy, and habits in need of improvement). The healthy pattern was characterized by a higher intake of water, vegetables, fish, fruit, healthy fat, oilseeds/avocado, lean meat, legumes, and low intake of sweetened beverages, sweets or commercially produced desserts, processed foods, and alcohol. The unhealthy pattern group frequently consumed sweetened beverages, foods not prepared at home, sweets or commercially produced desserts, processed foods, alcohol, and infrequently consumed water, vegetables, fish, and fruit. The group with habits in need of improvement frequently consumed unhealthy fat and whole grains and rarely consumed oilseeds/avocado, foods not prepared at home, and legumes. The analysis of variance showed differences in these patterns $(p<0.001)$ for each item of the questionnaire, and discriminant 
analysis confirmed that the dietary patterns had significant differences between them $(p<0.001)$, as was expected.

The analysis of dietary patterns (Table 4) identified that only $34.4 \%$ of seropositive cases report healthy food intake. In contrast, seronegative individuals present this same pattern in $38.1 \%$ of the cases. However, this difference was not significant $(p=0.221)$.

The individual food intake habits showed to be similar in both groups, except for the most consumed type of fat ( $p=0.006)$, since $26.9 \%$ of the seronegative cases and $20 \%$ of seropositive cases consume monounsaturated fats more frequently than other types of fats. Conversely, $69 \%$ of the seronegative individuals and $74.7 \%$ of those seropositive cases consume polyunsaturated fats more frequently. In both groups, the optimal consumption of vegetables, fish, legumes, oilseeds, and avocado is infrequent $(<15 \%)$.

Table 4. Food intake quality in individuals negative or positive to anti-SARS-CoV-2 antibodies 


\begin{tabular}{|c|c|c|c|c|}
\hline Question & Answer options & $\begin{array}{c}\text { Positive for } \\
\text { anti-SARS- } \\
\text { CoV-2 } \\
\text { antibodies* } \\
\text { N = 756 }\end{array}$ & $\begin{array}{c}\text { Negative } \\
\text { for anti- } \\
\text { SARS-CoV- } \\
2 \\
\text { antibodies } \\
\mathrm{N}=1043 \\
\end{array}$ & $\begin{array}{c}\text { P- } \\
\text { value }^{\mathrm{a}}\end{array}$ \\
\hline & & $\mathbf{N}(\%)$ & N (\%) & \\
\hline \multirow[t]{4}{*}{ 1. Do you drink at least 1.5 liters of water per day? } & A. Never & $16(2.1)$ & $23(2.2)$ & 0.141 \\
\hline & B. Sometimes & $237(31.3)$ & $285(27.3)$ & \\
\hline & C. Almost always & $276(36.5)$ & $373(35.8)$ & \\
\hline & D. Always & $227(30.0)$ & $362(34.7)$ & \\
\hline \multirow{4}{*}{$\begin{array}{l}\text { 2. Do you consume at least } 200 \text { grams of cooked or } \\
\text { raw vegetables per day? }\end{array}$} & A. Never & $17(2.2)$ & $35(3.4)$ & 0.104 \\
\hline & B. Sometimes & $376(49.7)$ & $466(44.7)$ & \\
\hline & C. Almost always & $257(34.0)$ & $396(38.0)$ & \\
\hline & D. Always & $106(14.0)$ & $146(14.0)$ & \\
\hline \multirow{4}{*}{$\begin{array}{l}\text { 3. Do you eat fresh or frozen fish }(100 \mathrm{~g}) \text { at least one } \\
\text { day per week? }\end{array}$} & A. Never & $122(16.1)$ & $146(14.0)$ & 0.351 \\
\hline & B. Sometimes & $452(59.8)$ & $641(61.5)$ & \\
\hline & C. Almost always & $118(15.6)$ & $181(17.4)$ & \\
\hline & D. Always & $64(8.5)$ & $75(7.2)$ & \\
\hline \multirow{4}{*}{$\begin{array}{l}\text { 4. Do you consume one or more glass (can) of } \\
\text { sweetened beverages per week? }\end{array}$} & A. Never & $116(15.3)$ & $193(18.5)$ & 0.319 \\
\hline & B. 1 to 3 times & $433(57.3)$ & $570(54.7)$ & \\
\hline & C. 4 to 6 times & $114(15.1)$ & $162(15.5)$ & \\
\hline & D. Daily & $93(12.3)$ & $118(11.3)$ & \\
\hline \multirow{4}{*}{$\begin{array}{l}\text { 5. Do you consume at least } 200 \text { grams of fruit per } \\
\text { day? }\end{array}$} & A. Never & $15(2.0)$ & $23(2.2)$ & 0.880 \\
\hline & B. Sometimes & $286(37.8)$ & 405 (38.8) & \\
\hline & C. Almost always & $294(38.9)$ & $408(39.1)$ & \\
\hline & D. Always & $161(21.3)$ & $207(19.1)$ & \\
\hline \multirow[t]{4}{*}{$\begin{array}{l}\text { 6. What type of fat do you consume most frequently on } \\
\text { a weekly basis? }\end{array}$} & $\begin{array}{c}\text { A. } \\
\text { Monounsaturated }\end{array}$ & $151(20.0)$ & $281(26.9)$ & 0.006 \\
\hline & $\begin{array}{c}\text { B. } \\
\text { Polyunsaturated }\end{array}$ & $565(74.7)$ & $720(69.0)$ & \\
\hline & C. Saturated & $23(3.0)$ & $23(2.2)$ & \\
\hline & D. Do not know & $17(2.2)$ & $19(1.8)$ & \\
\hline \multirow{4}{*}{$\begin{array}{l}\text { 7. Do you consume at least } 30 \text { grams of oilseeds or } \\
1 / 2 \text { of an avocado per day? }\end{array}$} & A. Never & $54(7.1)$ & $61(5.8)$ & 0.351 \\
\hline & B. Sometimes & $423(56.0)$ & $566(54.3)$ & \\
\hline & C. Almost always & $218(28.8)$ & $312(29.9)$ & \\
\hline & D. Always & $61(8.1)$ & $104(10.0)$ & \\
\hline \multirow{4}{*}{$\begin{array}{l}\text { 8. Do you eat foods not prepared at home three or } \\
\text { more days per week? }\end{array}$} & A. Never & $71(9.4)$ & $118(11.3)$ & 0.576 \\
\hline & B. Sometimes & $465(61.5)$ & $624(59.8)$ & \\
\hline & C. Almost always & $152(20.1)$ & $202(19.4)$ & \\
\hline & D. Always & $68(9.0)$ & $99(9.5)$ & \\
\hline \multirow[t]{3}{*}{ 9. What type of meat do you consume most often? } & A. Red meat & $399(52.8)$ & $516(49.5)$ & 0.361 \\
\hline & B. Chicken & $315(41.7)$ & $469(45.0)$ & \\
\hline & C. Fish & $42(5.6)$ & $58(5.6)$ & \\
\hline \multirow{4}{*}{$\begin{array}{l}\text { 10. Do you eat processed foods two or more days per } \\
\text { week? }\end{array}$} & A. Never & $112(14.8)$ & $129(12.4)$ & 0.398 \\
\hline & B. Sometimes & $567(75.0)$ & $792(75.9)$ & \\
\hline & C. Almost always & $70(9.3)$ & $112(10.7)$ & \\
\hline & D. Always & $7(0.9)$ & $10(1.0)$ & \\
\hline \multirow{3}{*}{$\begin{array}{l}\text { 11. Do you consume sweets or commercially produced } \\
\text { desserts two or more days per week? }\end{array}$} & A. Never & $64(8.5)$ & $105(10.1)$ & 0.470 \\
\hline & B. Sometimes & $542(71.7)$ & $719(68.9)$ & \\
\hline & C. Almost always & $120(15.9)$ & $182(17.4)$ & \\
\hline
\end{tabular}




\begin{tabular}{|c|c|c|c|c|}
\hline Question & Answer options & $\begin{array}{c}\text { Positive for } \\
\text { anti-SARS- } \\
\text { CoV-2 } \\
\text { antibodies* } \\
\text { N = } 756\end{array}$ & $\begin{array}{c}\text { Negative } \\
\text { for anti- } \\
\text { SARS-CoV- } \\
2 \\
\text { antibodies } \\
\mathrm{N}=1043\end{array}$ & $\begin{array}{c}\mathrm{P}- \\
\text { value }^{\mathrm{a}}\end{array}$ \\
\hline & & $\mathbf{N}(\%)$ & $\mathbf{N}(\%)$ & \\
\hline & D. Always & $30(4.0)$ & $37(3.5)$ & \\
\hline \multirow{4}{*}{$\begin{array}{l}\text { 12. Do you eat legumes at least three days per week } \\
\qquad(300 \mathrm{~g} \text { per week)? }\end{array}$} & A. Never & $20(2.6)$ & $37(3.5)$ & \multirow[t]{4}{*}{0.554} \\
\hline & B. Sometimes & $344(45.5)$ & $455(43.6)$ & \\
\hline & C. Almost always & $295(39.0)$ & $426(40.8)$ & \\
\hline & D. Always & $97(12.8)$ & $125(12.9)$ & \\
\hline \multirow[t]{3}{*}{ 13. What type of cereals do you consume most often? } & A. Whole grain & $260(34.4)$ & 406 (38.9) & \multirow[t]{3}{*}{0.145} \\
\hline & $\begin{array}{l}\text { B. Minimally } \\
\text { processed }\end{array}$ & $256(33.9)$ & $329(31.5)$ & \\
\hline & $\begin{array}{l}\text { C. Processed and } \\
\text { ultra-processed }\end{array}$ & $240(31.7)$ & $308(29.5)$ & \\
\hline \multirow{4}{*}{$\begin{array}{l}\text { 14. If you are a man, do you consume more than } 2 \\
\text { alcoholic beverages per day? If you are a woman, do } \\
\text { you consume more than } 1 \text { alcoholic beverage per day? }\end{array}$} & A. Never & $519(68.7)$ & $716(68.6)$ & \multirow[t]{4}{*}{0.077} \\
\hline & B. Sometimes & $228(30.2)$ & $296(28.4)$ & \\
\hline & C. Almost always & $8(1.1)$ & $26(2.5)$ & \\
\hline & D. Always & $1(0.1)$ & $5(0.5)$ & \\
\hline \multirow[t]{3}{*}{ Dietary patterns } & $\begin{array}{l}\text { Healthy food } \\
\text { intake }\end{array}$ & $260(34.4)$ & $397(38.1)$ & \multirow[t]{3}{*}{0.221} \\
\hline & $\begin{array}{l}\text { Habits in need of } \\
\text { improvement }\end{array}$ & 357 (47.2) & $453(43.4)$ & \\
\hline & $\begin{array}{l}\text { Unhealthy food } \\
\text { intake }\end{array}$ & 139 (18.4) & $193(18.5)$ & \\
\hline
\end{tabular}

*Anti-SARS-CoV-2 IgM, IgG, or IgM+ IgG seropositivity.

${ }^{a}$ Chi-square. Bold text indicates a statistically significant difference with a p-value less than 0.05 .

\section{Discussion}

Serological tests for SARS-CoV-2 are a topic of intense interest due to their potential to significantly enhance the diagnostic capability of healthcare services around the world in the current pandemic. However, as with all novel assays, each country should be specifically validating trials to understand the clinical relevance of results [16].

A recent study showed that COVID-19 patients, IgM and IgA antibodies to SARS-CoV-2 have been detected with a median of 5 (IQR, 3-6) days, while IgG was detected 14 (IQR, 10-18) days after symptom onset, with a positive rate of $85.4 \%, 92.7 \%$, and $77.9 \%$, respectively [17]. Moreover, this study concluded that the efficiency of antibody detection by IgM ELISA is higher than that of qPCR after 5.5 days of symptom onset. In our study, overall seropositive cases showed a median of 10 days with an IQR of 7-14 days. This range is greater than that previously reported by Li Guo et al., [17], which was taken as a cut-off point for the diagnosis algorithm applied in our study to define candidates for the serological tests. Based on 
this finding, we suggest that serological tests should be only recommended in patients with at least a week from the onset of symptoms This highlights the relevance to establish specific diagnosis algorithms for each population because there could be different kinetics for the development of antibodies, which could be affected by the interpretation of various factors such as the test used, the severity of the disease, or even genetic factors.

An earlier meta-analysis described no difference in the proportion of males and females infected with SARS-CoV-2 in several countries [18]. This does not appear to be the case in our study, as we found more seropositive cases in women. This result may be explained by the fact that individuals in this study were outpatients with mild or asymptomatic disease. It has been reported that the relative risk of severe COVID19 and dying from COVID-19 is higher for men than for women in almost all age groups worldwide [19]. Therefore, this could explain the overrepresented prevalence of women in our study cohort.

Regarding age, $27.03 \%$ of seropositive cases in this study were in the range of $21-30$ years old. These results reflect those of the Centers for Disease Control and Prevention (CDC), which reported in a recent analysis that in June 2020, COVID-19 incidence showed a rapid rate of increasing prevalence, and the highest overall incidence was among young adults [20]. This data is alarming since this population includes the social sector supporting the economy in most countries, including Mexico.

COVID-19 is characterized by many signs and symptoms shared with other infectious diseases [21]. In the present study, cough, respiratory distress, muscle and joint pain, and anosmia were the symptoms with higher prevalence in the seropositive cases than suspected cases that were seronegative. Therefore, we suggest that these symptoms should be considered as major criteria in decision-making to undergo a serological test for suspected COVID-19 and should also be considered major criteria to suggest the performance of a serological test for diagnosis of COVID-19. This finding applies to every anti-SARS-CoV2 antibody pattern studied ( $\operatorname{lgM}, \lg G$, or $\lg M+\lg G)$. One interesting finding is that anosmia was overrepresented in patients with the presence of IgM antibodies, thus, this symptom could be considered as markers of active disease.

COVID-19 prevalence is associated with the presence of comorbidities. A study in 22,757 COVID-19 patients from different countries reported that major comorbidities in the general population were hypertension $(27.4 \%)$, diabetes $(17.4 \%)$, cardiovascular disease (8.9\%), chronic obstructive pulmonary disease (7.5\%), cancer (3.5\%), and chronic kidney disease (2.6\%) [22]. Non-transmissible chronic disease is the main public health problem in Mexico; coronary heart disease and diabetes are the two of the leading causes of death in this country [23]. These population characteristics could be a significant factor in having a higher number of COVID-19 cases in Mexico. In the present study, we observed high comorbidities prevalence in seropositive and seronegative cases; however, diabetes was the only comorbidity more prevalent in seropositive cases than in seronegative cases. Thus, we propose this comorbidity as the major risk factor for COVID-19 in our population; in contrast, other countries such as China, South Korea, Italy, the USA, and the UK have reported hypertension as a major comorbidity [22]. This risk factor should also be analyzed in depth by the Mexican health authorities since previous studies 
support that this is a significant public health problem in this population and that it is a trigger for severe COVID-19, especially in people with social disadvantages and limited access to medical care [24].

As comorbidities, blood type and influenza vaccination status have been suggested as other risk factors for COVID-19. Previous studies showed that blood group A was associated with a higher risk for acquiring COVID-19 compared with non-A blood groups, whereas blood group 0 was associated with a lower risk for infection and mild disease $[8,9]$. The present study has been unable to replicate this finding; therefore, further studies are needed to clarify this discrepancy in other countries and verify ancestry as a potential confounding variable for this association.

Like that observed for blood type, this study did not show that individuals vaccinated against influenza had a lower risk of contracting COVID-19. In fact, 50.93\% of the positive cases reported having been vaccinated from the last quarter of 2019. This finding contrasts with results from other studies which reported influenza vaccination coverage rates that correlated negatively with SARS-CoV-2 seroprevalence [10], and that vaccination is associated with relative protection against COVID-19 [11]. Despite these discrepancies, we suggest that the high percentage of vaccination rate observed in the seropositive cases in this study could be associated with the moderate clinical manifestations that the patients presented since none of them had severe COVID-19. Therefore, we do not reject that influenza vaccination could be a protective factor for severe COVID-19, maybe by the previous proposed trained immunity mechanism [11].

Regarding the individual food intake habits, we observed a higher consumption of polyunsaturated fats in seropositive cases than in those seronegative. Polyunsaturated fatty acids are highly consumed by individuals because of their potential health benefits in chronic diseases. However, numerous studies have shown that these compounds are immunoregulatory and immunosuppressive, and thus may increase susceptibility to infection [25-27]. These findings should be much scrutinized, but this study highlights sustained conclusions for a new branch of COVID-19 susceptibility study.

A weakness of this study is that we did not follow up the entire clinical course and dynamic antibody seroconversion in seropositive cases; therefore, it was not possible to associate some variants with the severity of the disease or the clinical outcome. Thus, we could not obtain a confirmatory test by PCR to confirm possible false negatives in seronegative cases. Further research is required to fully understand the association of consumption of polyunsaturated fats as a probable risk factor of SARS-CoV-2 infection. Specifically, a scrutiny of the most consumed type of food and its consumption amounts is required.

\section{Conclusion}

In conclusion, due to the global emergency of COVID-19 and the insufficient infrastructure for molecular diagnosis in developing countries, there is a need to establish serological diagnostic testing to help diagnose the prevalence of COVID-19 in the population and detect individuals who were exposed to the virus. This study shows that antibody tests can be useful to detect COVID-19 patients, even in the acute 
phase. Also, it supports the association of diabetes as a principal risk factor for SARS-CoV-2 infection in the Mexican population. On the other hand, the results do not support nor reject previous associations between blood group or influenza vaccination as protective factors against SARS-CoV-2 infection. However, frequent consumption of polyunsaturated fats is highlighted as a new risk factor for COVID-19, which, as with all novel findings, should be corroborated by more studies.

\section{Abbreviations}

COVID-19: Coronavirus disease 2019

SARS-CoV-2: Severe acute respiratory syndrome coronavirus 2

IgM: Immunoglobulin M

IgG: Immunoglobulin G

RT-qPCR: Real-time polymerase chain reaction

Mini-ECCA: Mini-Survey to Evaluate Food Intake Quality

ANOVA: Analysis of variance

IQR: Interquartile range

ELISA: Enzyme-Linked Immunosorbent Assay

\section{Declarations}

\section{ACKNOWLEDGEMENTS}

The authors would like to thank all participants who cooperated with this study. We also especially thank Alonso López for their support in the graphic design of the surveys, Diego Alexis Jara for your help in grammar correction, Ernesto Alonso for your survey support and teamwork, and Ricardo Villanueva for your leadership in this project.

\section{AUTHORS' CONTRIBUTIONS}

M-O G and H-B J conceived of the presented idea; Y-T P, M-S AC, T-C R, L-R MG, G-A AC, and Z-N JS developed the sampling processes and survey application; V-M AN, G-CH M, and G-E G entered data and performed the statistical analysis; M-V JF, MG-O, and H-B J wrote the paper. All authors reviewed the paper and approved it.

\section{FUNDING}

Not Applicable. 


\section{AVAILABILITY OF DATA AND MATERIALS}

The data used and analyzed during the study are available from the corresponding author.

\section{ETHICS APPROVAL AND CONSENT TO PARTICIPATE}

Ethical approval for the project was provided by the Committee of Research, Ethics in Research and Biosecurity of the University Center of Health Sciences of the University of Guadalajara (No: Cl-07329). Written informed consent was obtained from the study participants or their parents.

\section{CONSENT FOR PUBLICATION}

Not Applicable.

\section{COMPETING INTERESTS}

The authors declare that they have no competing interests.

\section{References}

1. Chan JF-W, Yuan S, Kok K-H, To KK-W, Chu H, Yang J, et al. A familial cluster of pneumonia associated with the 2019 novel coronavirus indicating person-to-person transmission: a study of a family cluster. Lancet Lond Engl. 2020;395:514-23.

2. Rai P, Kumar BK, Deekshit VK, Karunasagar I, Karunasagar I. Detection technologies and recent developments in the diagnosis of COVID-19 infection. Appl Microbiol Biotechnol. 2021.

3. Paradiso AV, De Summa S, Loconsole D, Procacci V, Sallustio A, Centrone F, et al. Rapid Serological Assays and SARS-CoV-2 Real-Time Polymerase Chain Reaction Assays for the Detection of SARSCoV-2: Comparative Study. J Med Internet Res. 2020;22:e19152.

4. Deeks JJ, Dinnes J, Takwoingi Y, Davenport C, Spijker R, Taylor-Phillips S, et al. Antibody tests for identification of current and past infection with SARS-CoV-2. Cochrane Database Syst Rev. 2020;6:CD013652.

5. Long Q-X, Tang X-J, Shi Q-L, Li Q, Deng H-J, Yuan J, et al. Clinical and immunological assessment of asymptomatic SARS-CoV-2 infections. Nat Med. 2020;26:1200-4.

6. Muñiz-Diaz E, Llopis J, Parra R, Roig I, Ferrer G, Grifols J, et al. Relationship between the ABO blood group and COVID-19 susceptibility, severity and mortality in two cohorts of patients. Blood Transfus Trasfus Sangue. 2020.

7. Severe Covid-19 GWAS Group, Ellinghaus D, Degenhardt F, Bujanda L, Buti M, Albillos A, et al. Genomewide Association Study of Severe Covid-19 with Respiratory Failure. N Engl J Med. 2020;383:1522-34.

8. Zhao J, Yang Y, Huang H, Li D, Gu D, Lu X, et al. Relationship between the ABO Blood Group and the COVID-19 Susceptibility. medRxiv. 2020;:2020.03.11.20031096. 
9. Zietz M, Zucker J, Tatonetti NP. Associations between blood type and COVID-19 infection, intubation, and death. Nat Commun. 2020;11:5761.

10. Amato $M$, Werba JP, Frigerio B, Coggi D, Sansaro D, Ravani A, et al. Relationship between Influenza Vaccination Coverage Rate and COVID-19 Outbreak: An Italian Ecological Study. Vaccines. 2020;8.

11. Debisarun PA, Struycken P, Domínguez-Andrés J, Moorlag SJCFM, Taks E, Gössling KL, et al. The effect of influenza vaccination on trained immunity: impact on COVID-19. medRxiv. 2020;:2020.10.14.20212498.

12. Pawlowski C, Puranik A, Bandi H, Venkatakrishnan AJ, Agarwal V, Kennedy R, et al. Exploratory analysis of immunization records highlights decreased SARS-CoV-2 rates in individuals with recent non-COVID-19 vaccinations. medRxiv. 2020;:2020.07.27.20161976.

13. Salem ML, El-Hennawy D. The possible beneficial adjuvant effect of influenza vaccine to minimize the severity of COVID-19. Med Hypotheses. 2020;140:109752.

14. Naja F, Hamadeh R. Nutrition amid the COVID-19 pandemic: a multi-level framework for action. Eur J Clin Nutr. 2020;74:1117-21.

15. Bernal-Orozco MF, Badillo-Camacho N, Macedo-Ojeda G, González-Gómez M, Orozco-Gutiérrez JF, Prado-Arriaga RJ, et al. Design and Reproducibility of a Mini-Survey to Evaluate the Quality of Food Intake (Mini-ECCA) in a Mexican Population. Nutrients. 2018;10.

16. Bermingham WH, Wilding T, Beck S, Huissoon A. SARS-CoV-2 serology: Test, test, test, but interpret with caution! Clin Med. 2020;20:365-8.

17. Guo L, Ren L, Yang S, Xiao M, Chang D, Yang F, et al. Profiling Early Humoral Response to Diagnose Novel Coronavirus Disease (COVID-19). Clin Infect Dis Off Publ Infect Dis Soc Am. 2020. doi:10.1093/cid/ciaa310.

18. Peckham H, de Gruijter NM, Raine C, Radziszewska A, Ciurtin C, Wedderburn LR, et al. Male sex identified by global COVID-19 meta-analysis as a risk factor for death and ITU admission. Nat Commun. 2020;11:6317.

19. Ahrenfeldt LJ, Otavova M, Christensen K, Lindahl-Jacobsen R. Sex and age differences in COVID-19 mortality in Europe. Res Sq. 2020. doi:10.21203/rs.3.rs-61444/v1.

20. CDCMMWR. COVID-19 Stats: COVID-19 Incidence, by Age Group - United States, March 1November 14, 2020. MMWR Morb Mortal Wkly Rep. 2021;69. doi:10.15585/mmwr.mm695152a8.

21. Fu L, Wang B, Yuan T, Chen $X$, Ao Y, Fitzpatrick T, et al. Clinical characteristics of coronavirus disease 2019 (COVID-19) in China: A systematic review and meta-analysis. J Infect. 2020;80:656-65.

22. Bajgain KT, Badal S, Bajgain BB, Santana MJ. Prevalence of comorbidities among individuals with COVID-19: A rapid review of current literature. Am J Infect Control. 2020. doi:10.1016/j.ajic.2020.06.213.

23. Villalpando S, Rodrigo JR. The status of non-transmissible chronic disease in Mexico based on the National Health and Nutrition Survey 2006. Salud Pública México. 2010;52:S2-3. 
24. Sosa-Rubí SG, Seiglie JA, Chivardi C, Manne-Goehler J, Meigs JB, Wexler DJ, et al. Incremental Risk of Developing Severe COVID-19 Among Mexican Patients With Diabetes Attributed to Social and Health Care Access Disadvantages. Diabetes Care. 2020.

25. Jones GJB, Roper RL. The effects of diets enriched in omega-3 polyunsaturated fatty acids on systemic vaccinia virus infection. Sci Rep. 2017;7:15999.

26. Husson M-O, Ley D, Portal C, Gottrand M, Hueso T, Desseyn J-L, et al. Modulation of host defence against bacterial and viral infections by omega-3 polyunsaturated fatty acids. J Infect. 2016;73:52335 .

27. Schwerbrock NMJ, Karlsson EA, Shi Q, Sheridan PA, Beck MA. Fish oil-fed mice have impaired resistance to influenza infection. J Nutr. 2009;139:1588-94.

\section{Figures}

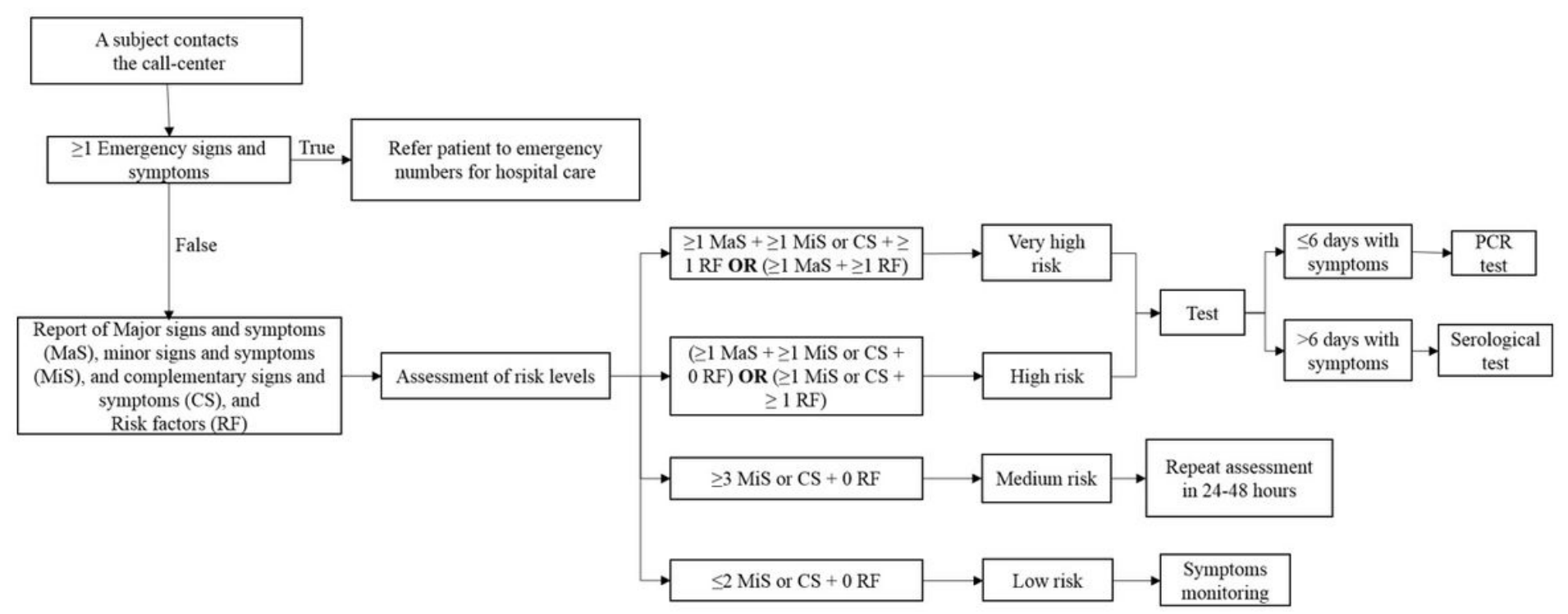

Figure 1

Algorithm to determine if a subject is a candidate for the test. Emergency signs and symptoms: difficulty breathing, bluish lips, chest pain, difficulty standing up, convulsions; major signs and symptoms (MaS): fever, dry cough, headache, irritability (children), loss of smell (anosmia), loss of taste (dysgeusia); minor signs and symptoms (MiS): shivers, muscular pain/soreness, bone pain, runny nose (rhinorrhea), sore throat, conjunctivitis; complementary signs and symptoms (CS): flu or cold, generally feeling unwell (malaise), tiredness/fatigue, cough with expectoration (phlegm), diarrhea, vomit, abdominal pain, fast breathing, nasal congestion; risk factors (RF): contact with suspected/confirmed COVID-19 case, healthcare worker, $>60$ years old, pregnancy, diabetes mellitus, hypertension, COPD, asthma, immunosuppression, HIV/AIDS, heart disease, obesity, kidney failure, smoking, cancer, liver failure, the time frame between symptoms appear and seeking medical care is $>5$ days. 